\title{
Leucine-rich protein 130 contributes to apoptosis resistance of human hepatocarcinoma cells
}

\author{
MICKAËL MICHAUD ${ }^{1,3}$, STÉPHANE BARAKAT ${ }^{1,4}$, SANDRINE MAGNARD ${ }^{1}$, \\ DOMINIQUE RIGAL ${ }^{2}$ and LORIS G. BAGGETTO ${ }^{1}$
}

\author{
${ }^{1}$ Institut de Biologie et Chimie des Protéines - UMR5086 CNRS UCBL, 7 Passage du Vercors, F-69367 Lyon Cedex 07; \\ ${ }^{2}$ Établissement Français du Sang Lyon, 1-3 Passage du Vercors, F-69007 Lyon, France
}

Received May 21, 2010; Accepted September 2, 2010

DOI: 10.3892/ijo_00000836

\begin{abstract}
LRP130 is a ubiquitous protein involved in cellular homeostasis, microtubule alteration, and transactivation of a few multidrug resistance genes. Its role in resistance to apoptosis in HepG2 and HUH7 hepatocarcinoma cells was investigated. Using shRNA-producing lentiviruses to downregulate the LRP130 gene, we showed that i) LRP130 did not affect the capacity of hepatocarcinoma cells to extrude drugs since LRP130 down-regulation was insufficient to significantly reduce $\mathrm{P}$-glycoprotein production in these cells, and ii) the expression of 11 apoptosis-related genes measured by PCRarray was significantly reduced. Interestingly, six of these genes encode extrinsic pathway proapoptotic proteins whose expression was higher in LRP130-non producing than in LRP130-producing HepG2 cells. Fluorescence microscopy confirmed this new anti-apoptotic role of LRP130, which is strengthened by a significantly reduced cytochrome $c$ oxidase activity in LRP130-down-regulated hepatocarcinoma cells.
\end{abstract}

Correspondence to: Dr Loris G. Baggetto, IBCP UMR5086 CNRS, 7 Passage du Vercors, F-69367 Lyon cedex 07, France

E-mail: lg.baggetto@ibcp.fr

Present addresses: ${ }^{3}$ Institut Gustave Roussy, INSERM, U848, 94805 Villejuif, France; ${ }^{4}$ IRIC, C.P. 6128, succursale Centre-ville, Montréal QC H3C 3J7, Canada

Abbreviations: BCRP, breast cancer resistance protein; COX, cytochrome $c$ oxidase; Em, emission wavelength; Ex, excitation wavelength; FACS, fluorescence-activated cell sorter; GFP, green fluorescent protein; hnRNPK, heterogeneous nuclear ribonucleoprotein complex; LRP130, Leucine-rich protein 130; LSFC, LeighSyndrome French-Canadian type; MDR, multidrug resistance; MOI, multiplicity of infection; MRP1, multi-drug resistance associated protein1; MVP, major vault protein; PGC-1 $\alpha$, peroxisome proliferatorsactivated receptor coactivator $1 \alpha$; P-gp, P-glycoprotein; RT-qPCR, reverse-transcription quantitative PCR; shRNA, short-hairpin RNA; siRNA, small interfering RNA

Key words: apoptosis, multidrug resistance, lentiviral vector, Leucine-Rich protein 130, siRNA

\section{Introduction}

Leucine-Rich protein 130 (LRP130 or LRPPRC) (1-3) contains 11 pentatricopeptide repeat motifs supposed to bind hydrophilic structures such as nucleotides $(4,5)$, and preferably localizes in the nucleus, mitochondria, or near the nuclear envelope (6-9).

Although little is known about this protein, LRP130 appears to be involved in several human or murine metabolic processes. Human LRP130 binds mitochondrial and nuclear RNAs, and is a component of the hnRNPK complex for RNA nuclear export $(6,10)$. Studies on murine LRP130 supported its implication in RNA export, linked this protein to the minisatellite DNA stability, and evidenced a binding domain for single-stranded DNAs and RNAs $(7,11)$. We previously reported that human LRP130 could bind double-stranded DNA (12). Furthermore, LRP130 is a component of several protein complexes $(4,13,14)$, and may play different roles concerning cytoskeleton organization, vesicular transport, mitochondrial metabolism, chromosome activity, and apoptosis. Additionally, LRP130 plays a central metabolic role since it participates in the formation of the transcriptional activator PGC-1 $\alpha$ involved in liver glucose homeostasis, energy metabolism, and nuclear receptor activation (15). Finally, a mutation (A3354V) in the LRP130 sequence is responsible for the Leigh Syndrome, French-Canadian type (LSFC), a neurodegenerative disorder that results from cytochrome $c$ deficiency in brain and liver $(16,17)$ in which LRP130 affects gluconeogenesis and electronic flow in the respiratory chain.

Cancer multidrug resistance (MDR) phenotype (reviewed in ref. 18) is composed of both typical and atypical aspects. Typical MDR is conferred by the overexpression of membrane transporters such as P-glycoprotein (ABCB1), MRP1 (ABCC1), or BCRP (ABCG2), which actively extrude drugs out of the cell. Atypical MDR regroups several cellular mechanisms that could notably affect drug inactivation, resistance to apoptosis, or DNA repair systems, among other processes (19-21). We previously showed that LRP130 transactivated the P-glycoprotein-encoding MDRl gene, strongly suggesting its participation in the typical MDR phenotype.

In this study, we used RNA interference to further study the implication of LRP130 in cancer cell chemoresistance. 
We tested the capacity of hepatocarcinoma cells to extrude drugs according to the LRP130 expression status. Moreover, since LRP130 appears to play a role in several key metabolic pathways, its role in chemoresistance could involve other mechanisms than its transcriptional activity on MDR-related genes. Notably, a link between LRP130 and the apoptotic pathways has repeatedly been evoked $(6,11,13)$ without being further studied. Since resistance to apoptosis is an important MDR mechanism, we investigated the influence of LRP130 on the expression of several apoptosis-related genes. The results obtained have been compared with in vitro experiments to demonstrate that LRP130 actually contributes to the atypical MDR phenotype by delaying the entry of HepG2 cells to apoptosis.

\section{Materials and methods}

Chemicals. All reagents were from Sigma-France, unless otherwise indicated.

Cell culture. Human hepatocarcinoma cell lines HepG2 and HUH7 were cultured in RPMI-1640 and DMEM, respectively, at $37^{\circ} \mathrm{C}$ in water-saturated atmosphere and $5 \% \mathrm{CO}_{2}$. In both cases the culture medium was supplemented with $10 \%$ heatinactivated bovine serum albumin, $100 \mathrm{U} / \mathrm{ml}$ penicillin, $100 \mathrm{mg} / \mathrm{ml}$ streptomycin, and $0.25 \mathrm{mg} / \mathrm{ml}$ amphotericin B. Cell viability was regularly checked by trypan blue exclusion.

Transfection. Cells were transfected with 2 siRNAs targeting the LRP130 mRNA: si(LRP130)a (5'-GGAGGUCUAUGA AUAUAAA-3') designed and supplied by Qiagen, and si(LRP130)b designed and supplied by Ambion (code ID 36416). Both were prepared and transfected into cells by using Hi-perfect (Qiagen), according to the manufacturer's instructions. Unless indicated, cells reaching 50-80\% confluency were transfected by $25 \mathrm{nM}$ of siRNA and serum media was not replaced until analysis $48 \mathrm{~h}$ later. A control siRNA ('All Star Negative Control siRNA', Qiagen) was used for each experiment.

Production of lentiviral particles carrying a shRNA. The lentiviral vector FG12 (22) was used to carry 2 siRNA sequences targeting LRP130 mRNA: si(LRP130)1 (5'-acc GGATCTCCCAGTTACAGAGttcaagagaCTCTGTAACTG GGAGATCCttttc-3') and si(LRP130)2: (5'-accGGAGGU CUAUGAAUAUAAAttcaagagaTTTATATTCATAGACCT CCttttc- $\left.3^{\prime}\right)$; the siRNA sequence is in capital letters. The control shRNA sequence with no known target was 5'-acc GAAAAACCAACCGGTTAGGttcaagagaCCTAACCGGTT GGTTTTTCtttttc-3'. These sequences were first placed downstream a HuU6 promoter of a pBS-HuU6 vector within Bbs 1 and Xho1 restriction sites, the HuU6/shRNA cassette being further inserted into the FG12 lentiviral vector between the $X b a 1$ and $X h o 1$ restriction sites. To produce viral particles, $2.4 \times 10^{6} 293 \mathrm{~T}$ cells cultured in DMEM were seeded in a Petri dish on the first day, and transfected on the second day by the classical $\mathrm{CaCl}_{2}$ method with $5 \mu \mathrm{g}$ of DNA containing $2.7 \mu \mathrm{g}$ envelope-coding plasmid VSV-G $(9 \mathrm{~kb}), 9 \mu \mathrm{g}$ SIV3+ GAG POL TAT REV plasmid $(14 \mu \mathrm{g})$ and $9 \mu \mathrm{g}$ FG12 vector plasmid $(7-8 \mathrm{~kb})$. The supernatant carrying viral particles was collected $72 \mathrm{~h}$ after transfection and impurities were eliminated by clarification (centrifugation at $300 \mathrm{x} \mathrm{g}$ ) and filtration. It was finally titrated before direct use or stockpiling at $-80^{\circ} \mathrm{C}$. The viral titer was determined by using 293T cells as reference, which were infected by increasing volumes of virus-containing supernatant. The percentage of cells effectively infected, which produced GFP, was assessed by flow cytometry (FACsort, Beckton-Dickinson FACS system) using the CellQuest analysis software (Beckton-Dickinson, FACS system). An estimation of the titer is given by the ratio (number of GFP+ cells)/ (number of infected cells) when the vector is not saturating.

Cell infection with lentiviral particles. HepG2 and HUH7 cells $\left(3 \times 10^{5}\right)$ were seeded in 6 -well plates on the first day, washed twice $32 \mathrm{~h}$ later with PBS 1X, and pre-incubated for $20 \mathrm{~min}$ in their respective culture medium supplemented with $16 \mu 1 / \mathrm{ml}$ polybrene. Supernatants containing viral particles were directly added to the cell medium, so that approximately $3 \times 10^{6}$ particles could infect cells $(\mathrm{MOI}=10)$. Fifteen hours post-infection, cells were meticulously washed 3 times with PBS $1 \mathrm{X}$ and allowed to grow in a polybrene-free medium for indicated times until analysis.

Protein expression analysis. HepG2 or HUH7 cells $\left(3 \times 10^{6}\right)$ in a 6-well plate were lysed with Laemmli buffer $1 \mathrm{X}(62.5 \mathrm{mM}$ Tris-HCl pH 6.8; $10 \%$ glycerol; 2\% w/v SDS; 5\% ß-merceptoethanol; $0.0062 \% \mathrm{w} / \mathrm{v}$ bromophenol blue). Protein concentration was determined using 'DC Protein Assay' (Bio-Rad), before separation of $40 \mu \mathrm{g}$ proteins by SDS-PAGE, and electrotransfer at $250 \mathrm{~mA}$ during $90 \mathrm{~min}$ onto a nitrocellulose membrane (Bio-Rad) at $4^{\circ} \mathrm{C}$ in the following buffer: $10 \%$ methanol, $25 \mathrm{mM}$ Tris-base, $192 \mathrm{mM}$ glycine, $\mathrm{pH}$ 8.3. The nitrocellulose membrane was incubated for at least $45 \mathrm{~min}$ under mild agitation in 5\% skimmed milk (Régilait) dissolved in TBS-Tween buffer $(150 \mathrm{mM} \mathrm{NaCl}, 20 \mathrm{mM}$ Tris $\mathrm{HCl}$, Tween-20 0.1\%, pH 7.4). The membrane was then washed and incubated either at $4^{\circ} \mathrm{C}$ overnight in the presence of polyclonal anti-LRP130 (Eurogentec) or monoclonal C219 (Dako) antibodies, or at $37^{\circ} \mathrm{C}$ for $1 \mathrm{~h}$ with mouse monoclonal antiP53 or anti- $\beta$-actin (Sigma) antibodies in TBS-Tween containing 3\% BSA (Sigma) and $0.02 \%$ sodium azide. The second peroxidase-labelled anti-mouse (Sigma) or anti-rabbit (Promega) antibodies were incubated for $90 \mathrm{~min}$ at room temperature in TBS-Tween with 5\% skimmed milk, and the reaction was revealed by chemiluminescence using ECL Western Blotting Detection Reagents (Amersham Biosciences).

Drug retention test. Cells $\left(1 \times 10^{4}\right)$ were washed with PBS $1 \mathrm{X}$, and incubated with $1.9 \mu \mathrm{M}$ daunomycin $(\mathrm{Ex}=520 \mathrm{~nm}, \mathrm{Em}=$ $596 \mathrm{~nm}$ ) for $3 \mathrm{~h}$ in culture medium and at $37^{\circ} \mathrm{C}$. They were further washed twice with PBS $1 \mathrm{X}$, trypsinized, washed again with PBS $1 \mathrm{X}$ and homogenized in $100 \mu \mathrm{l}$ of ice-cold PBS $1 \mathrm{X}$. Daunomycin fluorescence was detected in each sample by flow cytometry and the drug retention profiles obtained were analyzed using CellQuest.

Gene expression analysis. Total RNAs from HepG2 or HUH7 cells $\left(1 \times 10^{4}\right.$ to $\left.1 \times 10^{6}\right)$ were extracted using the RNeasy Mini kit (Qiagen), quantified using a Nanodrop ND-100 spectrophotometer, and $100 \mathrm{ng}$ were retrotranscribed by RT-AMV 
(Promega). RT-qPCR was realized in Thermo-Fast 96-well PCR (AB gene) in the presence of SYBR green. We used the following primers (Sigma Genosys): 5'-ATGGACGTTATT GCCAGGAG-3' and 5'-GCTCAACCAACAAGTGAGCA-3' for LRP130 (NM_133259), 5'-CAACCGCGAGAAGATGA CCC-3' and 5'-CAGAGGCGTACAGGGATAGCA-3' for Bactin (NM_001101), 5'-GGAACTTTCCGGAATGACAA-3' and 5'-GTCACTCCAGGGCGTACAAT-3' for TNFSF10 (NM_003844), 5'-AAGCAAACCTCGGGGATACT-3' and 5'-TGCATCCAAGTGTGTTCCAT-3' for caspase-8 (NM_001228), 5'-CCGTATCCATCGAAGCAGAT-3' and 5'-GTGGCCAGACCAAGTAGGAA-3' for caspase-10 (NM_001230), 5'-GGTTCATCCAGTCGCTTTGT-3' and 5'-AATTCTGTTGCCACCTTTCG-3' for caspase-3 (NM_4346). The results obtained were collected and analyzed with the iCycler software (Bio-Rad), and the relative quantity of each gene was normalized to that of the reference $\beta$-actin gene. A standard curve to assess efficacy was realized.

PCR-array. The 'Human apoptosis' $\mathrm{RT}^{2}$ Profiler PCR Array (SA Bioscience Corporation) was used to compare the expression levels of 84 apoptosis-related genes in LRP130expressing (LRP130+) or not (LRP130-) apoptotic HepG2 cells. Total RNAs $(1 \mu \mathrm{g})$ were converted to cDNA and used as templates. Values obtained were collected with the 'I-cycler' software and compared gene by gene among different samples using the manufacturer's array available at http://www. superarray.com/PCRArrayPlate.php, which normalizes values between 2 plates using 5 internal reference genes for each plate.

Test of entrance into apoptosis. Cells were seeded on a cover glass to reach $30-70 \%$ confluency at observation time. Apoptosis was induced by incubating cells with $2 \mu \mathrm{l} / \mathrm{ml}$ antiFAS antibody for $24 \mathrm{~h}$ at $37^{\circ} \mathrm{C}$. Afterwards, cells were washed twice with PBS $1 \mathrm{X}$ and incubated in culture medium containing $6.8 \mu \mathrm{M} \mathrm{JC}-1$ (to assess mitochondrial potential) and $10 \mu \mathrm{M}$ Hoechst 33342 (to assess pyknosis) for $30 \mathrm{~min}$ at $37^{\circ} \mathrm{C}$ and in the dark. Finally, cells were washed twice with PBS $1 \mathrm{X}$ and observed by fluorescence microscopy (Axioplan 2, Zeiss). Image acquisition was realized through a FITC filter using a Cool Snap camera (Photometrics) and METAVUE software. For FACS analysis, cells were seeded on a 6 well-plate and apoptosis was induced as previously described. Then, approximately $1 \times 10^{6}$ cells were washed twice with PBS $1 \mathrm{X}$ and incubated in a buffer containing Annexin V (Annexin-V Fluos Staining kit, Roche) and propidium iodide for $15 \mathrm{~min}$ in the dark and at room temperature. The emitted fluorescence was detected by flow cytometry and analyzed with CellQuest.

Isolation of mitochondria, cytochrome oxidase (COX) activity, and protein measurement. HepG 2 or HUH7 cells $\left(2 \times 10^{6}\right)$ were collected with a cell lifter and pelleted by centrifugation at $1,000 \mathrm{x}$ g. Isolation of mitochondria was performed using the MitoProfile Benchtop Mitochondria Isolation kit for Cultured Cells (MitoSciences).

The COX activity of isolated mitochondria, normalized to the activity of citrate synthase (23), was measured photometrically as described (24). Briefly, mitochondria were solubilized in assay buffer $(50 \mathrm{mM}$ potassium phosphate buffer, $\mathrm{pH} 7.2,5 \%$ (v/v) lubrol). Ferrous cytochrome $c$ was prepared by reduction with dithiotreitol and purified by desalting on a PP-10 column (GE Healthcare). The COX reaction was started by addition of ferrous cytochrome $c$ (final concentration $120 \mu \mathrm{M}$ ) and the initial rate of oxidation was determined at room temperature by following the decrease in absorbance at $550 \mathrm{~nm}$. Protein concentration was measured by the Bradford method using fatty acid-free serum albumin as a standard (25).

\section{Results}

LRP130 down-regulation in hepatocarcinoma cells using shRNA-carrying lentiviral vectors. We have previously shown that LRP130 was implicated in the typical MDR phenotype due to its transcriptional activity on MDR-related genes such as MDRI (12). Inhibition of the LRP130 production by RNA interference appears to be a promising strategy, but the effects of the classical method for transient transfection of siRNAs appeared neither to be continuous nor to last long enough to observe both an extinction of the LRP130 protein and a resulting effect on MDR related-genes expression. Therefore, to assess the possibility of permanently inhibiting LRP130 expression, we used an shRNA-carrying lentiviral vector. Two shRNA sequences were cloned in the FG12 plasmid as described previously (22). Viral particles were successfully produced and infection of targeted cells was carried out using optimal parameters reported in Materials and Methods. Fluorescence microscopy (Fig. 1A) shows that HepG2 and HUH7 cells could be fully infected. Moreover, cells exhibited their usual shape, growth rate, and morphology, indicating that the infection did not induce any serious morphologic or metabolic anomaly. The Table in Fig. 1A reports the capacity of shRNA-carrying lentiviral vectors to infect HepG2 and HUH7 cells when different viral charges were tested on each cell line. The good infectivity of this vector is illustrated by the effective infection of 37.8 to $49.9 \%$ of cells when an MOI of 1 was achieved. Results also indicate that a MOI of 10 seemed optimal in order to perform other experiments as this was sufficient to infect $92.1 \% \pm 4.1$ of HepG2 cells and $85.6 \% \pm 4.7$ of $\mathrm{HUH7}$ cells.

In order to evaluate the efficiency of the shRNA sequences to down-regulate the LRP130 gene, we controlled the production of LRP130 and the expression of its mRNA by Western blotting and RT-qPCR, respectively. Fig. 1B shows that both si(LRP130)1 and si(LRP130)2 sequences almost completely cancelled both LRP130 production and the expression of its gene in both cell lines compared to the control, thereby validating the use of such lentiviral vectors for RNA interference by both shRNAs in hepatocarcinoma cells. Therefore, lentiviral particles successfully and permanently inhibited LRP130 expression in HEPG2 and HUH7 hepatocarcinoma cells.

The contribution of LRP130 to cell resistance relating to drug extrusion is not significant in hepatocarcinoma cells. Stable inhibition of LRP130 was assessed by its role on the capacity of HepG2 and HUH7 cells to extrude drugs. Due to LRP130 transactivating role on the MDRl gene, LRP130 extinction should potentially decrease the amount of cellular P-gp, and 


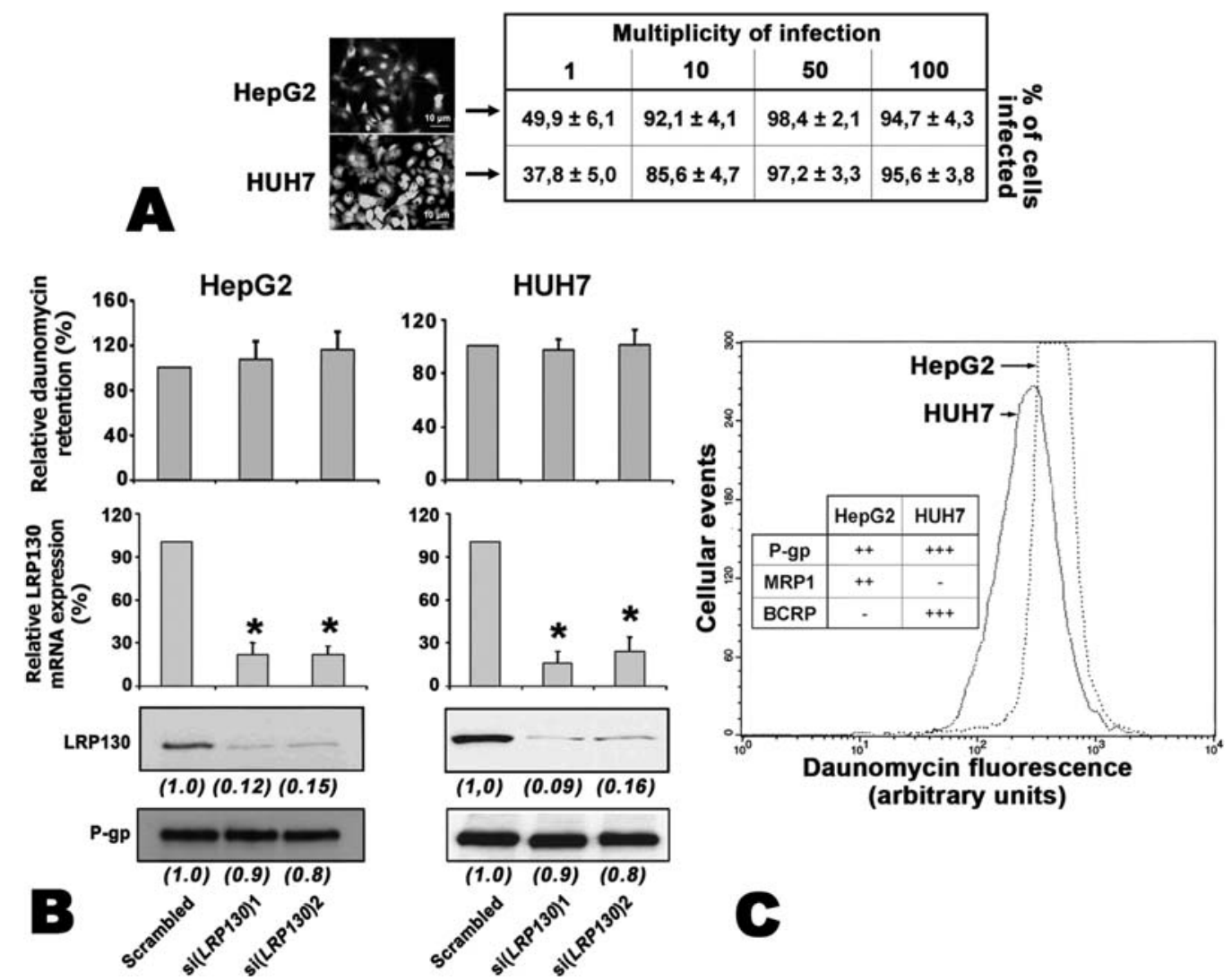

Figure 1. Inhibition of LRP130 expression by lentiviral-mediated RNA interference and its consequence on drug extrusion in HepG2 and HUH7 cell lines. (A) Infectious capacity of viral particles encoding both GFP and LRP130 shRNAs. HepG2 and HUH7 cells $\left(1 \times 10^{6}\right)$ were infected with different MOIs, and analyzed for GFP expression by flow cytometry $96 \mathrm{~h}$ later. Results are means of 3 independent experiments. A MOI of 10 represented optimal conditions for further experiments and was used to obtain fluorescence microscopy images. (B) Efficiency of the shRNAs targeting LRP130 mRNA and their influence on P-gp and LRP130 production and daunomycin retention. Two lentivirus-carried shRNA sequences [si(LRP130)1 and si(LRP130)2] were tested in each cell line and compared with a scrambled sequence with no known target (scrambled). Intracellular accumulation of daunomycin was measured by FACS in 3 independent experiments and is given relatively to the control. To measure the expression of LRP130 messenger (2nd raw), 2x10 cells were infected and total RNAs were extracted 3 days later. LRP130 expression was assessed by RT-qPCR. Results of 3 independent experiments are given relatively to the control. Asterisks represent a statistically significant variation (t-test, $\mathrm{p}<0.05$ ). To assess LRP130 and P-gp production (3rd raw), $1 \times 10^{6}$ cells were infected, lysed 4 or 5 days later, and analyzed by Western blot analysis with polyclonal anti-LRP130 or monoclonal C219 antibodies, respectively. A control for the stable production of $\beta$-actin as reference was realized (not shown). Numbers between parentheses represent band intensities [measured with the ImageJ (NIH) software] relative that of the control. (C) Daunomycin retention profile of HepG2 and HUH7 cells obtained by FACS. Cells (2x105) were infected and incubated 5 days later for $3 \mathrm{~h}$ in the presence of $1.9 \mu \mathrm{M}$ daunomycin before analysis by flow cytometry. Estimated relative quantification of P-gp, MRP1, and BCRP transporters in each cell lines, carried out by RT-PCR and Western blot analysis, is indicated in the insert.

thus the cell resistance conferred by this protein. Fig. 1C shows the intracellular retention of daunomycin by HepG2 and HUH7 cells and their relative amount of P-glycoprotein, MRP1 and BCRP drug transporters (insert) as estimated by Western blot analysis. We observed that the amounts of P-gp and BCRP were high in HUH7 cells, whereas HepG2 cells produced average amounts of P-gp and MRP1. These results underline the fact that several transporters were co-produced by these hepatocarcinoma cell lines, many of which had previously been identified as responsible for the typical MDR phenotype. Moreover, Fig. 1C strongly suggests that HUH7 cells displayed a better ability to extrude daunomycin than did HepG2 cells. To assess the role of LRP130 on daunomycin extrusion, the retention of this drug was measured in cells previously infected by viral particles carrying si(LRP130)1, si(LRP130)2, or a control sequence. Fig. 1B shows no significant difference in daunomycin extrusion, whether the cells expressed LRP130 or not, thereby excluding the possibility that LRP130 itself could be responsible for daunomycin extrusion. The same experiment was carried out with mitoxantrone in place of daunomycin and similar results were obtained (data not shown). In addition, a Western blot analysis (Fig. 1B) shows that LRP130 down-regulation slightly affected the production of P-gp with a corresponding slightly increased daunomycin retention in HepG2. This result supports our former data since the expected decreased production of P-gp due to LRP130 down-regulation should have modified the daunomycin retention profile. Nonetheless, the fact that the amount of P-gp was slightly affected by the absence of LRP130 remains surprising and will be discussed later. These results strongly suggest that LRP130 does not seem to play a significant role in the typical MDR phenotype of hepatocarcinoma cells.

Evidence for the involvement of LRP130 in resistance to apoptotic stimuli by its control on the expression of apoptoticrelated genes. Since apoptotic and MDR mechanisms are tightly correlated in cancer cells, we wondered whether 
Table I. Differences in apoptosis-related gene expression between LRP130+ and LRP130- apoptotic HepG2 cells.

\begin{tabular}{|c|c|c|c|c|c|}
\hline Protein symbol ${ }^{\mathrm{a}}$ & $\begin{array}{c}\text { GeneBank } \\
\text { sequence } \\
\text { number }\end{array}$ & $\begin{array}{c}\text { Fold } \\
\text { difference }\end{array}$ & Protein symbol & $\begin{array}{c}\text { GeneBank } \\
\text { sequence } \\
\text { number }\end{array}$ & $\begin{array}{c}\text { Fold } \\
\text { difference }\end{array}$ \\
\hline ABL1 [11] & NM_005157 & -2.08 & CASPASE8 [4,9] & NM_001228 & 6.23 \\
\hline AKT1 $[11,12]$ & NM_005163 & -2.75 & CD40 [2] & NM_001250 & -1.87 \\
\hline BAD $[3,11]$ & NM_004322 & -2.09 & CD40LG [1] & NM_000074 & -1.95 \\
\hline BAG3 $[3,12]$ & NM_004281 & 2.06 & CIDEA [10] & NM_001279 & -2.08 \\
\hline BAG4 [3] & NM_004874 & 3.84 & CRADD [7] & NM_003805 & 2.53 \\
\hline $\mathrm{BAX}[3,11]$ & NM_004324 & 4.72 & DFFA [10] & NM_004401 & 2.71 \\
\hline BCL2 $[3,11,12]$ & NM_000633 & -2.23 & FADD $[8,9]$ & NM_003824 & 1.90 \\
\hline BCL-XL/S $[3,11,12]$ & NM_138578 & 3.84 & LTA $[1]$ & NM_000595 & -2.95 \\
\hline BCL2L $10[3,12]$ & NM_020396 & -3.16 & MCL1 $[3,12]$ & NM_021960 & 4.72 \\
\hline BCL2L11 [3] & NM_006538 & -2.08 & NOL3 [7] & NM_003946 & -2.57 \\
\hline BCL2L2 $[3,12]$ & NM_004050 & -2.08 & PYCARD [7] & NM_013258 & -3.39 \\
\hline BFAR [12] & NM_016561 & 2.20 & TNFRSF10B $[2,8]$ & NM_003842 & 7.67 \\
\hline $\mathrm{BID}[3,11]$ & NM_001196 & 2.31 & TNFRSF11B $[2,8]$ & NM_002546 & -2.08 \\
\hline BIK [3] & NM_001197 & 2.91 & TNFRSF1A $[2,8]$ & NM_001065 & -2.08 \\
\hline BIRC3 $[5,7,12]$ & NM_001165 & 2.95 & TNFRSF7 (CD27) [2] & NM_001242 & -3.84 \\
\hline BIRC6 $[5,12]$ & NM_016252 & 3.58 & TNFRSF9 [2] & NM_001561 & -2.01 \\
\hline BIRC8 $[5,12]$ & NM_033341 & -2.08 & TNFSF10 [1] & NM_003810 & -2.00 \\
\hline CARD4 (NOD1) [7] & NM_006092 & -1.96 & TNFSF7 (CD70) [1] & NM_001252 & -3.63 \\
\hline CASPASE10 $[4,9]$ & NM_001230 & 4.11 & TNFSF8 [1] & NM_001244 & -2.57 \\
\hline CASPASE14 [4] & NM_012114 & -2.08 & TP53 [11] & NM_000546 & 4.72 \\
\hline CASPASE2 $[4,7]$ & NM_032982 & 3.84 & TP53BP2 [11] & NM_005426 & -2.08 \\
\hline CASPASE4 $[4,7]$ & NM_001225 & -2.57 & TP73 [11] & NM_005427 & -1.92 \\
\hline CASPASE5 $[4,7]$ & NM_004347 & -2.08 & TRAF4 [6] & NM_004295 & 4.72 \\
\hline CASPASE7 $[4,11]$ & NM_001227 & 2.56 & HPRT1 & NM_000194 & -1.95 \\
\hline
\end{tabular}

Expression level of each of the 48 reported genes was assessed by 'PCR-array', as described in Fig. 2. A variation was considered positive

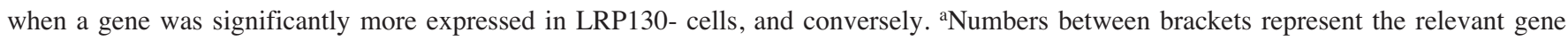
family of the listed protein: [1], TNF ligand; [2], TNF receptor; [3], Bcl-2; [4], Caspase; [5], IAP; [6], TRAF; [7], CARD; [8], death domain; [9], death effector domain; [10], CIDE domain; [11], p53 and DNA damage response; [12], anti-apoptosis.

LRP130 could influence the MDR phenotype by affecting the pro- or anti-apoptotic pathways. In such a case, LRP130 would be directly associated with the atypical MDR phenotype. In order to investigate this hypothesis, we compared the expression of apoptosis-related genes in LRP130-down regulated (LRP130-) or not (LRP130+) HepG2 cells. Preliminary results with the $\mathrm{RT}^{2}$ profiler PCR array technology showed that variations in apoptosis-related gene expression were small (data not shown); however, they strongly suggested that many apoptotic genes from the extrinsic pathway were better expressed in LRP130- than in LRP130+ cells. To evidence such an observation, the same experiment was carried out in cells in which apoptosis had previously been induced. If several apoptotic genes were actually more expressed, the difference in expression levels obtained between the LRP130+ and LRP130- samples would be expected to be greater. Apoptosis was triggered by incubating cells with $2 \mu \mathrm{l} / \mathrm{ml}$ anti-FAS antibody in conditions that induced a significant increase $(30 \%)$ of the number of apoptotic cells in a sample, as revealed by flow cytometry (data not shown). A PCR-array using HepG2 apoptotic cells showed significant variations in the expression levels of apoptosis-related genes between LRP130- and LRP130+ cells (Table I), contrasting with the results obtained with non-apoptotic cells, thereby suggesting that LRP130 actually was involved in this process. Expression of eleven of the studied genes coding for BCL- $\mathrm{X}_{\mathrm{L} / \mathrm{S}}$, caspase-2, caspase-8, caspase-10, BAX, TP53, TNFRSF10, TNFSF7, TRAF-4, BAG-4, and MCL-1, proved to vary significantly as shown in Fig. 2A and B. The expression of TNFRSF10 and caspase- 8 genes was the most affected by the absence of LRP130 as its level increased by a $7.67 \pm 1.4$ and $6.23 \pm 1.1$ factor, respectively. All these proteins are depicted in Fig. 2B, which shows that most of them are pro-apoptotic, among which 6 are directly implicated in the extrinsic apoptotic pathway. These results were further corroborated by RT-qPCR on selected genes (Fig. 2C), since in the absence of LRP130 the expression of caspase-8, -10, -3, and TNFRSF10 genes was respectively $6.1,5.2,3.3$, and 8.0 -fold greater than that measured in its presence. Finally, a confirming Western blot analysis (Fig. 2D) shows that production of P53 doubled in response to an apoptotic stimulus on (LRP130-) cells.

All these results show that LRP130 represses the expression of many pro-apoptotic genes and proteins involved in the extrinsic apoptotic pathway in response to an apoptotic stimulus, and strongly suggest that LRP130 plays an antiapoptotic role in hepatocarcinoma cells. 


A
\begin{tabular}{|c|c|c|}
\hline $\begin{array}{c}\text { Protein } \\
\text { symbol }\end{array}$ & Reference & $\begin{array}{c}\text { Fold } \\
\text { difference }\end{array}$ \\
\hline BAG-4 & NM_004874 & $3,84 \pm 1,2$ \\
\hline BAX & NM_004324 & $4,72 \pm 0,9$ \\
\hline BCL-X Ls & NM_138578 & $3,84 \pm 1,3$ \\
\hline Caspase 10 & NM_001230 & $4,11 \pm 1,1$ \\
\hline Caspase 2 & NM_032982 & $3,84 \pm 1,0$ \\
\hline Caspase 8 & NM_001228 & $6,23 \pm 1,1$ \\
\hline MCL-1 & NM_021960 & $4,72 \pm 1,6$ \\
\hline TNFSF 10a & NM_001242 & $7,67 \pm 1,4$ \\
\hline TNFSF 7 & NM_003844 & $-3,8 \pm 1,0$ \\
\hline TP53 & NM_000546 & $4,72 \pm 1,0$ \\
\hline TRAF-4 & NM_004295 & $4,72 \pm 1,2$ \\
\hline
\end{tabular}
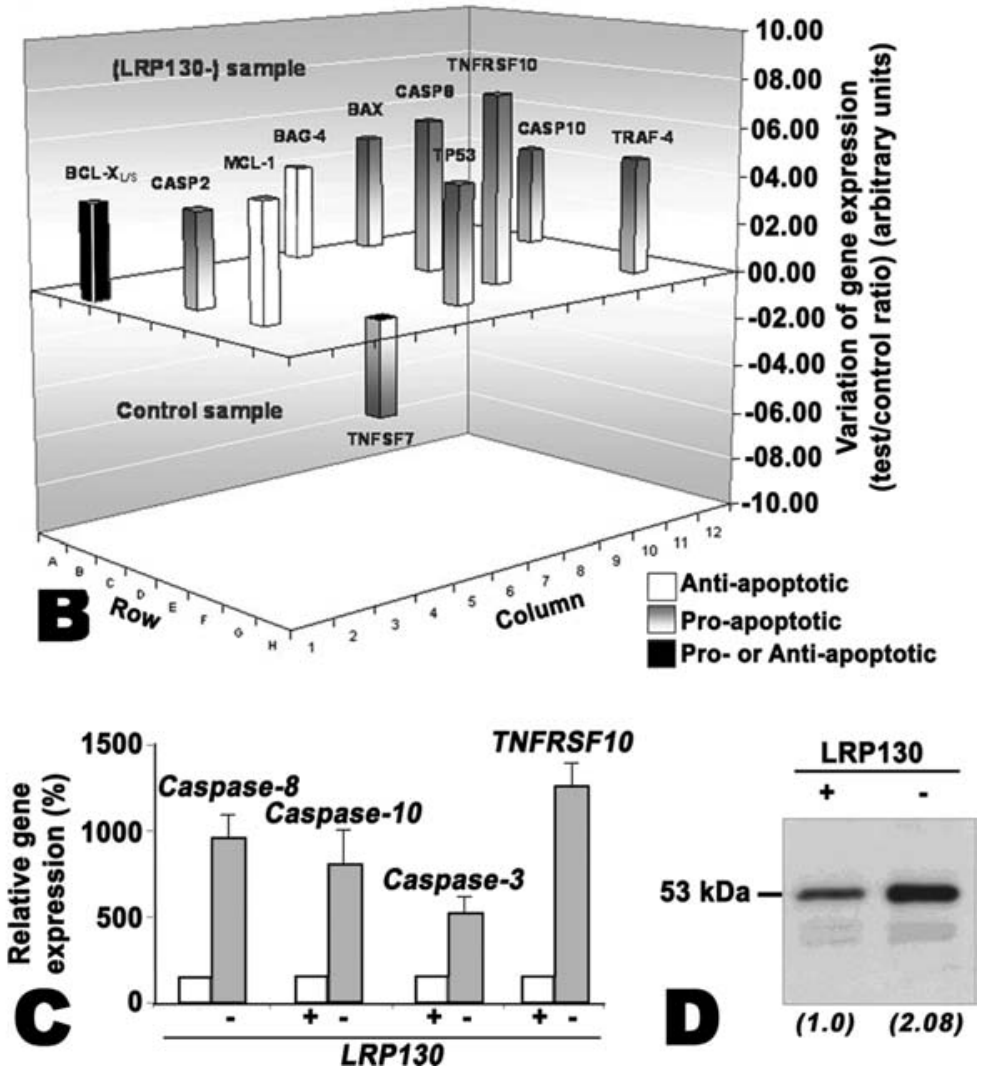

Figure 2. Influence of LRP130 on apoptosis-related gene expression in apoptotic HepG2 cells. Analysis of gene expression and their product was carried out in LRP130+/control and LRP130- cells and treated with anti-FAS antibody to trigger apoptosis. (A) Variation in apoptosis-related gene expression measured with Human Apoptosis RT ${ }^{2}$ Profiler PCR Array. LRP130+/control and LRP130- cells $\left(1 \times 10^{6}\right)$ were incubated for $24 \mathrm{~h}$ with $2 \mu 1 / \mathrm{ml}$ anti-FAS antibody. A variation was considered positive when a gene was significantly more expressed in LRP130- cells, and conversely. Only selected genes, the variation of which was superior to a 3.5 factor, were reported here. Data are the result of 3 independent experiments. (B) Illustration of (A). Each column represents a gene mentioned in (A). (C) Relative expression of caspase-3, -8, -10, and TNFRSF10 genes in LRP130+ and LRP130- apoptotic cells analyzed by RT-qPCR. Templates were identical to those used in (A). Values are given relatively to those obtained from LRP130+ samples and are means of 3 independent experiments. (D) Production of the TP53 protein in LRP130+ and LRP130- apoptotic cells observed by Western blot analysis. Cells were incubated for $24 \mathrm{~h}$ with $2 \mu \mathrm{l} / \mathrm{ml}$ anti-FAS antibody, then lysed and analyzed in the presence of monoclonal anti-P53 antibody. Expression of the reference protein $\beta$-actin as a control was realized, but is not shown here. Numbers between parentheses represent band intensities relative to the control.

LRP130 delays the entry to apoptosis of HepG2 cells in vitro and directly contributes to the atypical MDR phenotype. The role of LRP130 in the apoptotic pathways was assessed by comparing the entrance into apoptosis of LRP130+ and LRP130- HepG2 cells previously exposed to an apoptotic stimulus with the use of several fluorescent probes. However, owing to the possible interference with probe detection of GFP produced by the lentiviral vector used to inhibit LRP130, we had rather used synthetic siRNAs designed according to the sequences that were successfully tested with the lentiviral vector (Fig. 1C). Experimental conditions were optimized by taking into account the overproduction of LRP130 as well as the difficulty to transfect HepG2 cells, so that significant levels of inhibition of LRP130 production with the si(LRP130)a sequence could be reached, as shown in Fig. 3A. Fig. 3A1 shows LRP130 gene expression observed by RT-PCR at 24, 48, 72 and $96 \mathrm{~h}$ after siRNA transfection. With the conditions recommended by the manufacturer ( $25 \mathrm{nM}$ siRNA), LRP130 gene expression was inhibited by no more than $30 \%$, and quickly regained its control value. A higher siRNA concentration $(100 \mathrm{nM})$ seemed inefficient as well since gene expression only decreased by $50 \%$, which was not enough to observe any significant decrease in protein production (data not shown). Interestingly, incubation of HepG2 cells with 3 consecutive additions of $100 \mathrm{nM}$ siRNA every $24 \mathrm{~h}$ induced a strong and sustained inhibition of the LRP130 gene (Fig. 3A1) and an almost total absence of the protein after $72 \mathrm{~h}$ (Fig. 3A2). siRNA concentration and stability appeared to be determining factors for the efficiency of RNA interference in this cell line. Nevertheless, this result underlines the great potentiality of synthetic siRNAs even in case of the inhibition of an overexpressed protein, or the targeting of hard-to-transfect cell lines. Moreover, it allowed us to compare the apoptotic response between LRP130+ and LRP130- cells. Entrance of cells into apoptosis was assessed by flow cytometry using Annexin-V and propidium iodide labeling (Fig. 3B1). The results obtained were analyzed, and are reported in Fig. 3B2. In the presence of $2 \mu \mathrm{l} / \mathrm{ml}$ anti-FAS antibody for $24 \mathrm{~h}$, the apoptotic population of HepG2 cells increased 2.8 times as much as that of the control, while the amount of the necrotic population remained stable. However, in the absence of LRP130, the apoptotic population increased 4.1 times as much as that of the control, suggesting that LRP130 delayed or prevented the entry of 

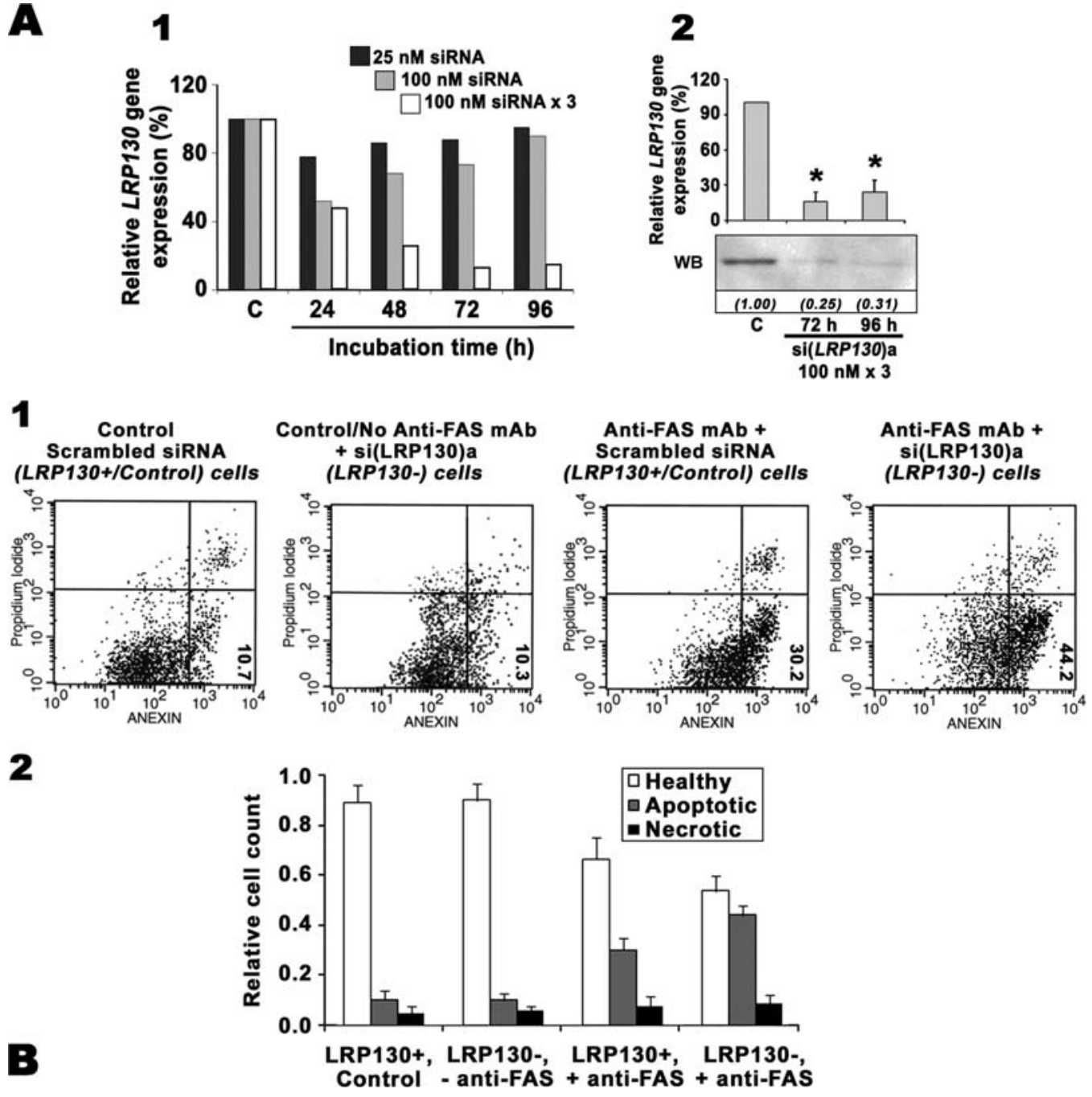

Figure 3. Comparison of cell death sensitivity between HepG2 cells expressing LRP130 (LRP130+) or not (LRP130-). (A) LRP130 silencing in HepG2 cells using synthetic siRNA. [1] Cells $\left(2 \times 10^{5}\right)$ were transfected by $25 \mathrm{nM}, 100 \mathrm{nM}$, or 3x100 nM (100 nM every 24 h) of si(LRP130)a siRNA, and LRP130 mRNA expression was determined by RT-qPCR after 24, 48, 72 and $96 \mathrm{~h}$ of incubation time. Results are given relatively to a control sample treated by a scrambled sequence with no known target (C). One typical experiment is presented. [2] Cells were transfected with $100 \mathrm{nM}$ si(LRP130)a every 24 h, then tested for LRP130 production and its gene expression 72 and $96 \mathrm{~h}$ later, as in the legend of Fig. 1C. Numbers between parentheses represent band intensities relative to the control. Results are means of 3 independent experiments and are given relatively to a control sample transfected with a scrambled sequence with no known target (C). Asterisks represent a statistically significant variation (t-test, $\mathrm{p}<0.05)$. (B) Flow cytometry detection of induced cell death in LRP130+ and LRP130- HepG2 cells. [1] Cells were transfected either by a scrambled siRNA sequence (LRP130+/control cells), or by the si(LRP130)a sequence (LRP130- cells). When needed, $1 \times 10^{5}$ cells were incubated for $24 \mathrm{~h}$ with anti-FAS antibody. FACS analysis was carried out using 'Annexin-V Fluos Staining kit' (Roche). The percentage of apoptotic cells is reported in the lower-right quadrant of each cytogram. One typical experiment is presented. [2] Graphical representation of the results reported in Fig. 3B1. For each sample, the number of healthy, apoptotic and necrotic cells is given relatively to the total number of analyzed cells. Results are the means of 3 independent experiments.

HepG2 cells to apoptosis in response to an apoptotic stimulus. A control in the absence of both LRP130 and anti-FAS antibody showed no significant difference with the LRP130+ control.

The fluorescent probes JC-1 and Hoechst 33342 were used to assess the mitochondrial potential and the condensed status of chromatin, respectively. Fig. 4 shows that the apoptotic state is clearly more advanced in LRP130- than in LRP130+ HepG2 cells when exposed to anti-FAS antibodies. A higher number of LRP130+ cells could be observed (due to a smaller number of disrupted cells); their mitochondrial potential was higher, while the number of pyknotic nuclei was 3 to 4 times lower than those of the LRP130- population. Conversely, a result similar to the control was obtained in the absence of both anti-FAS antibody and LRP130.
The human apoptosis PCR array we used did not include genes involved in oxidative metabolism. One of the major consequences of apoptosis stimulation would be a decreased oxidative metabolism as suggested by the result obtained in Fig. 4B showing that when apoptosis was induced by antiFAS antibodies, LRP130- cells exhibited a marked decrease of their mitochondrial transmembrane electrochemical potential when compared to LRP130+ cells. To assess this observation, we measured the specific activity of the cytochrome $c$ oxidase enzyme marker on permeabilized mitochondria isolated from LRP130+ and LRP130- cells. Fig. 5 shows that when apoptosis was induced by anti-FAS antibodies, the specific activity of COX decreased by $88.2 \%$ when compared to the control whereas it was only reduced by $35 \%$ in the presence of LRP130, thereby suggesting the protective role of LRP130 on the 


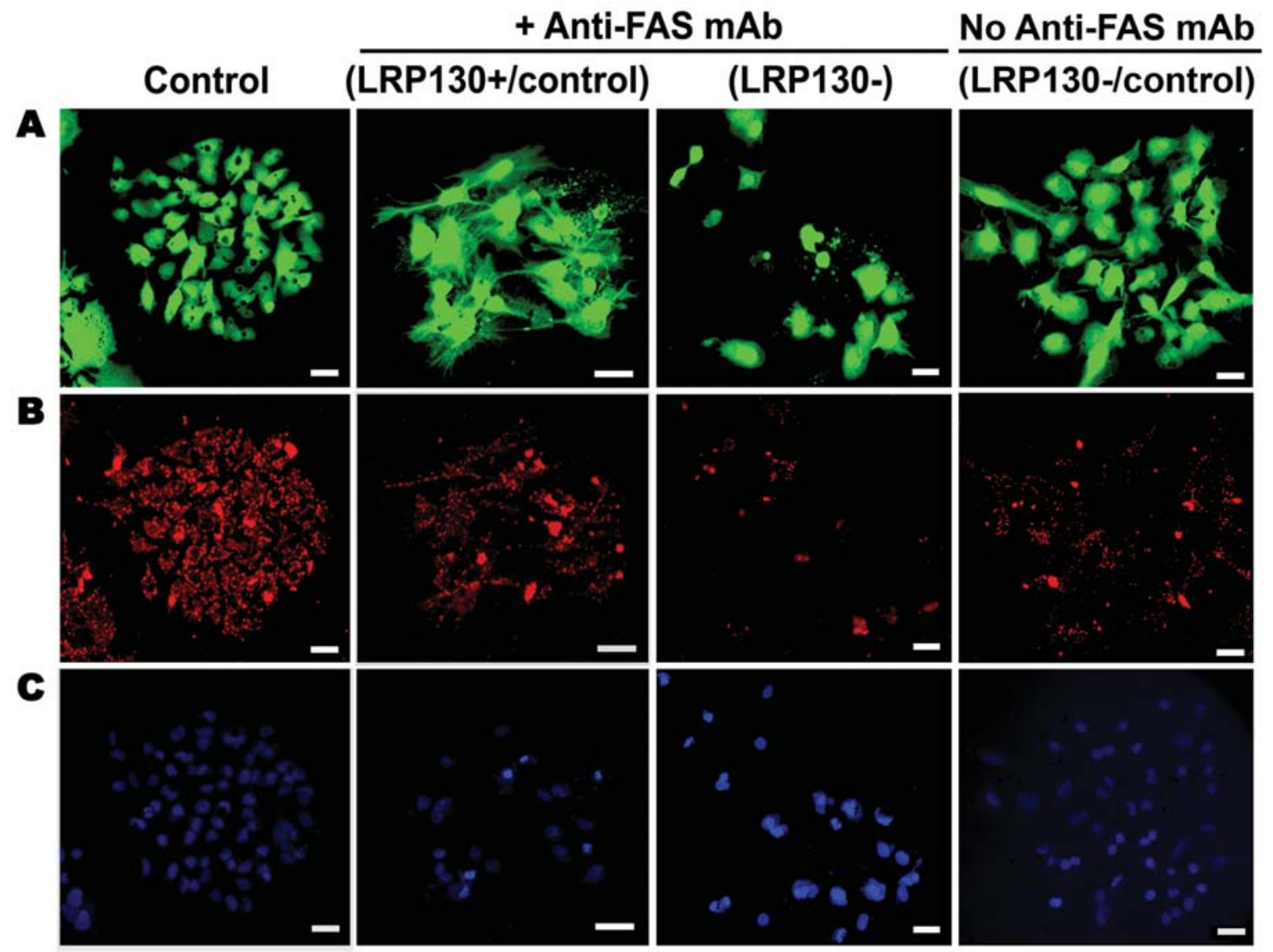

Figure 4. Detection of apoptosis by fluorescence microscopy in LRP130+ and LRP130- HepG2 cells. Cells were transfected either by a scrambled siRNA (LRP130+/control), or by the si(LRP130)a sequence (LRP130-), as described in the legend of Fig. 3A. When needed, apoptosis was induced by incubating cells with anti-FAS antibody for $24 \mathrm{~h}$. All cells were washed and incubated with $6.8 \mu \mathrm{M}$ JC-1 and $10 \mu \mathrm{M}$ Hoechst 33342 during 30 min, and observed by fluorescence microscopy. (A) Cell adherence to the substratum. (B) Mitochondrial potential evaluated by the JC-1 probe; the acquisition time was the same for each sample (500 ms). (C) Pyknotic nuclei evidenced by Hoechst 33342. Scale bar represents $5 \mu \mathrm{m}$. One typical experiment is presented.

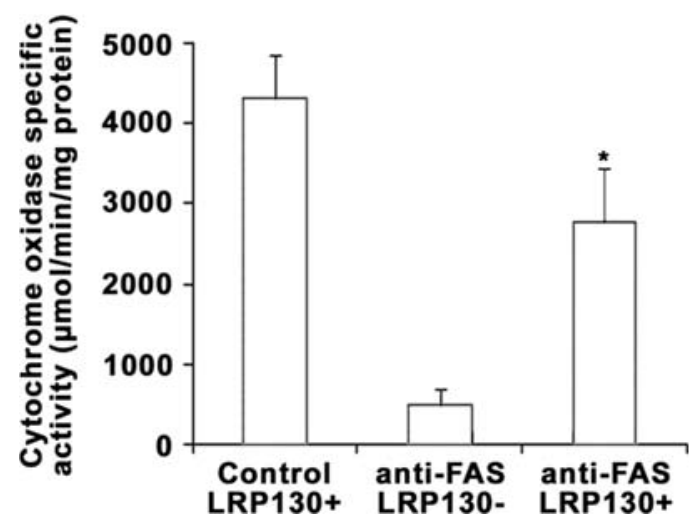

Figure 5. Specific activity of cytochrome $c$ oxidase of mitochondria isolated from control and LRP130-producing or not apoptotic cells. Except for controls, LRP130+ and LRP130- cells were incubated with anti-FAS antibodies prior to mitochondria isolation and permeabilization with $5 \%$ lubrol. Results are means of 3 independent experiments \pm standard error of the mean. The asterisks represent statistically significant variations (t-test, $\mathrm{p}<0.005)$.

oxidative metabolism. All these results strongly suggest that LRP130 plays an anti-apoptotic role in HepG2 cells, thereby indicating its contribution to the atypical MDR phenotype.

\section{Discussion}

LRP130 appears to have several different functions in cells. We previously reported that LRP130 could transactivate the MDRl and MVP genes (12), two main actors of the MDR phenotype (26). Moreover, other groups suggested a probable interaction of LRP130 with some actors of the apoptotic pathways $(13,14)$. Since apoptosis is involved in chemoresistance mechanisms, we hypothesized that LRP130 could participate in the MDR phenotype through both its transcriptional activity on MDR-related genes (12) and its modulation of cancer cell sensitivity to apoptosis. We used RNA interference to compare LRP130-expressing (LRP130+) or not (LRP130-) hepatocarcinoma cells (Fig. 1B and 3A), whose lines were chosen for their high level of LRP130 production (2), which would easily reveal its role in apoptosis when its production is inhibited.

We first observed that LRP130 inhibition induced a very light change in the cell's capacity to extrude drugs (Fig. 1C). This result was not expected since we previously showed that down-regulation of the LRPI30 gene induced a partial inhibition of the MDRl gene expression (12). Actually, the absence of LRP130 should have been accompanied with an important decreased cellular amount of P-gp, and de facto the daunomycin retention profile of these cells should have been 
been strongly altered, but this did not occur. However, our previous study was conducted using HT1080 fibrosarcoma cells whose behavior could greatly differ from HUH7 and HepG2 hepatocarcinoma cells. For instance, we observed that P-gp production was much lower in HT1080 than in HUH7 cells; we and others also observed that the type of transporters that are produced as well as their respective contribution to the MDR phenotype vary from one cell line to another (data not shown). Moreover, LRP130 is a transactivator of the MDR1 gene (12) among many other genes that have already been studied (27) and its inhibition alone should not suffice to significantly decrease the amount of P-gp. In addition, our previous study reported that only a small percentage of the LRP130 protein could be sufficient to maintain its transcriptional activity. These three arguments should be considered to explain why the amount of P-gp did not decrease to a high extent when LRP130 expression was inhibited. Therefore, we conclude that LRP130 does not play a significant role in drug extrusion by hepatocarcinoma cells since its presence does not seem to significantly modify the amount of P-gp, and does not significantly alter the intracellular drug accumulation either.

Our data also suggest that LRP130 plays a role in cell entry to apoptosis. The fact that P-gp production was not highly modulated in HepG2 cells in response to LRP130 inhibition facilitated this study since contradicting results were reported concerning the involvement of $\mathrm{P}$-gp itself in apoptotic pathways (28-30). Results in Table I and Fig. 2 show that the expression of pro-apoptotic genes was higher in LRP130- than in LRP130+ apoptotic HepG2 cells. These results were confirmed in vitro by observing that the absence of LRP130 facilitated the entry of the studied cells to apoptosis (Figs. 3B1, B2 and 4). These data seem to contrast with previous studies that reported the interaction between LRP130 and C19ORF5 in both the nucleus and the cytoplasm $(13,14)$. C19ORF5 induces cell death by accumulating on hyper-stabilized microtubules, and/or by interacting with the tumor suppressor RASSF1, thereby leading to the expectation of a pro-apoptotic action of LRP130. However, these authors only reported the LRP130/ C19ORF5 interaction, but not the role of the LRP130 protein in this complex. In the same manner, they proposed that LRP130 associates with the ubiquitously expressed transcript (UXT) whose accumulation can lead to apoptosis; meanwhile, the role of LRP130 in this complex remains unknown, in which it may act as a regulator for instance (31). Other studies underlined the importance of LRP130 in the mitochondrial metabolism that is responsible for its involvement in the Leigh-Syndrome, French-Canadian type $(3,15,32)$. These data clearly support our results since a deficient mitochondrial metabolism expectedly enhances the cellular sensitivity to apoptotic signals as well as cell entry to apoptosis. The PCR array we used did not include nuclear and mitochondrial genes involved in oxidative metabolism. This is why we measured the cytochrome $c$ oxidase (COX1) activity, which is an important enzyme marker of the inner mitochondrial membrane centrally involved in oxidative metabolism. Since COX1 specific activity was much more strongly depressed in LRP130- than in LRP130+ cells in which apoptosis was induced by anti-FAS antibodies, our results corroborate the role LRP130 plays in mitochondrial oxidative metabolism (LRP130 was mainly localized in mitochondria and nuclei of both HepG2 and HUH7 cells, results not shown). It is interesting to note that Pet309, a yeast homolog to the human LRP130, is involved in the translation of yeast mitochondrial $C O X 1$ gene encoding subunit 1 of cytochrome $c$ oxidase (33). Since COX1 activity is greatly reduced in LRP130- cells, LRP130 may also be involved in COX1 mRNA translation and/or stability in hepatocarcinoma cells.

In addition, several studies described the involvement of LRP130 in RNA stability and transport, thereby supporting its implication in cell viability $(6,10)$. This is also in accordance with another study that describes the up-regulation of the rLRP157rat ortholog of LRP130 during cell growth phases and supports its involvement in maintaining the phenotype via mRNA stabilization and transport (8). Although a direct role of LRP130 in the apoptotic pathways could not be established, our study attests of the active participation of this protein in the atypical MDR phenotype.

Since a few transcription factor-encoding genes like Runx3 have already been reported as modulators of both several apoptotic- and MDR-related gene expression (34), we cannot exclude that a transcriptional activity of LRP130 could also be responsible for its involvement in apoptosis resistance.

In conclusion, in addition to its previously reported role in cancer chemoresistance by transactivating MDR genes, we showed that LRP130 plays a new role in the MDR phenotype by delaying the entry of hepatocarcinoma cells to apoptosis and preserving mitochondrial oxidative metabolism. Although more work seems necessary to elucidate its precise anti-apoptotic mechanisms, LRP130 appears as an important molecular link between different MDR processes and should be considered as a promising therapeutic target.

\section{Acknowledgements}

We wish to thank Professor D. Baltimore for his kind gift of the FG12 vector, Dr Didier Nègre for viral particles production, and Mrs. Jeanine Bernaud for her help with the flow cytometry technique. This study was supported by grants from Ligue contre le Cancer (Comité du Rhône, Comité de la Loire, Comité de la Saône et Loire, Comité National). M.M. was a grant recipient from the Ligue contre le cancer, Comité de la Loire.

\section{References}

1. Ghiso NS and Lennon GG: $\operatorname{lrp} 130$ gene assigned to chromosome 2. In Vitro Cell Dev Biol Anim 30A: 744, 1994.

2. Hou J, Wang F and McKeehan WL: Molecular cloning and expression of the gene for a major leucine-rich protein from human hepatoblastoma cells (HepG2). In Vitro Cell Dev Biol Anim 30A: 111-114, 1994.

3. Mootha VK, Lepage P, Miller K, et al: Identification of a gene causing human cytochrome c oxidase deficiency by integrative genomics. Proc Natl Acad Sci USA 100: 605-610, 2003.

4. Liu L and McKeehan WL: Sequence analysis of LRPPRC and its SEC1 domain interaction partners suggests roles in cytoskeletal organization, vesicular trafficking, nucleocytosolic shuttling, and chromosome activity. Genomics 79: 124-136, 2002.

5. Small ID and Peeters N: The PPR motif - a TPR-related motif prevalent in plant organellar proteins. Trends Biochem Sci 25: 46-47, 2000. 
6. Mili S and Pinol-Roma S: LRP130, a pentatricopeptide motif protein with a noncanonical RNA-binding domain, is bound in vivo to mitochondrial and nuclear RNAs. Mol Cell Biol 23: 4972-4982, 2003.

7. Tsuchiya N, Fukuda H, Nakashima K, Nagao M, Sugimura T and Nakagama H: LRP130, a single-stranded DNA/RNA-binding protein, localizes at the outer nuclear and endoplasmic reticulum membrane, and interacts with mRNA in vivo. Biochem Biophys Res Commun 317: 736-743, 2004.

8. Eilers H, Trilk SL, Lee SY, et al: Isolation of an mRNA binding protein homologue that is expressed in nociceptors. Eur J Neurosc 20: 2283-2293, 2004.

9. Zeng $\mathrm{H}$ and Saari JT: Increased type I collagen content and DNA binding activity of a single-stranded, cytosine-rich sequence in the high-salt buffer protein extract of the copper-deficient rat heart. J Nutr Biochem 15: 694-699, 2004.

10. Mili S, Shu HJ, Zhao Y and Pinol-Roma S: Distinct RNP complexes of shuttling hnRNP proteins with pre-mRNA and mRNA: candidate intermediates in formation and export of mRNA. Mol Cell Biol 21: 7307-7319, 2001.

11. Tsuchiya N, Fukuda H, Sugimura T, Nagao M and Nakagama H: LRP130, a protein containing nine pentatricopeptide repeat motifs, interacts with a single-stranded cytosine-rich sequence of mouse hypervariable minisatellite Pc-1. Eur J Biochem 269: 2927-2933, 2002.

12. Labialle S, Dayan G, Gayet L, Rigal D, Gambrelle J and Baggetto LG: New invMED1 element cis-activates human multidrug-related MDR1 and MVP genes, involving the LRP130 protein. Nucleic Acids Res 32: 3864-3876, 2004.

13. Liu L, Amy V, Liu G and McKeehan WL: Novel complex integrating mitochondria and the microtubular cytoskeleton with chromosome remodeling and tumor suppressor RASSF1 deduced by in silico homology analysis, interaction cloning in yeast, and colocalization in cultured cells. In Vitro Cell Dev Biol Anim 38: 582-594, 2002

14. Liu L, Vo A, Liu G and McKeehan WL: Putative tumor suppressor RASSF1 interactive protein and cell death inducer C19ORF5 is a DNA binding protein. Biochem Biophys Res Commun 332: 670-676, 2005.

15. Cooper MP, Qu L, Rohas LM, et al: Defects in energy homeostasis in Leigh syndrome French Canadian variant through PGC-1alpha/LRP130 complex. Genes Dev 20: 2996-3009, 2006.

16. Merante F, Petrova-Benedict R, MacKay N, et al: A biochemically distinct form of cytochrome oxidase (COX) deficiency in the Saguenay-Lac-Saint-Jean region of Quebec. Am J Hum Genet 53: 481-487, 1993.

17. Morin C, Mitchell G, Larochelle J, et al: Clinical, metabolic, and genetic aspects of cytochrome C oxidase deficiency in SaguenayLac-Saint-Jean. Am J Hum Genet 53: 488-496, 1993.

18. Szakacs G, Paterson JK, Ludwig JA, Booth-Genthe C and Gottesman MM: Targeting multidrug resistance in cancer. Nat Rev Drug Discov 5: 219-234, 2006.

19. Sheweita SA: Drug-metabolizing enzymes: mechanisms and functions. Curr Drug Metab 1: 107-132, 2000.

20. El-Deiry WS: The role of p53 in chemosensitivity and radiosensitivity. Oncogene 22: 7486-7495, 2003.
21. Middleton MR and Margison GP: Improvement of chemotherapy efficacy by inactivation of a DNA-repair pathway. Lancet Oncol 4: 37-44, 2003.

22. Qin XF, An DS, Chen IS and Baltimore D: Inhibiting HIV-1 infection in human $\mathrm{T}$ cells by lentiviral-mediated delivery of small interfering RNA against CCR5. Proc Natl Acad Sci USA 100: 183-188, 2003.

23. Rustin P, Chretien D, Bourgeron T, et al: Biochemical and molecular investigations in respiratory chain deficiencies. Clin Chim Acta 228: 35-51, 1994.

24. Tzagoloff A, Akai A, Needleman RB and Zulch G: Assembly of the mitochondrial membrane system. Cytoplasmic mutants of Saccharomyces cerevisiae with lesions in enzymes of the respiratory chain and in the mitochondrial ATPase. J Biol Chem 250: 8236-8242, 1975.

25. Bradford MM: A rapid and sensitive method for the quantitation of microgram quantities of protein utilizing the principle of protein-dye binding. Anal Biochem 72: 248-254, 1976.

26. Gros P, Ben Neriah YB, Croop JM and Housman DE: Isolation and expression of a complementary DNA that confers multidrug resistance. Nature 323: 728-731, 1986.

27. Scotto KW: Transcriptional regulation of ABC drug transporters. Oncogene 22: 7496-7511, 2003.

28. Robinson LJ, Roberts WK, Ling TT, Lamming D, Sternberg SS and Roepe PD: Human MDR 1 protein overexpression delays the apoptotic cascade in Chinese hamster ovary fibroblasts. Biochemistry 36: 11169-11178, 1997.

29. Ruefli AA, Bernhard D, Tainton KM, Kofler R, Smyth MJ and Johnstone RW: Suberoylanilide hydroxamic acid (SAHA) overcomes multidrug resistance and induces cell death in $\mathrm{P}$ glycoprotein-expressing cells. Int J Cancer 99: 292-298, 2002.

30. Park SJ, Wu CH, Choi MR, Najafi F, Emami A and Safa AR: Pglycoprotein enhances TRAIL-triggered apoptosis in multidrug resistant cancer cells by interacting with the death receptor DR5. Biochem Pharmacol 72: 293-307, 2006.

31. Moss TN, Vo A, McKeehan WL and Liu L: UXT (ubiquitously expressed transcript) causes mitochondrial aggregation. In Vitro Cell Dev Biol Anim 43: 139-146, 2007.

32. Xu F, Morin C, Mitchell G, Ackerley C and Robinson BH: The role of the LRPPRC (leucine-rich pentatricopeptide repeat cassette) gene in cytochrome oxidase assembly: mutation causes lowered levels of COX (cytochrome c oxidase) I and COX III mRNA. Biochem J 382: 331-336, 2004.

33. Tavares-Carreon F, Camacho-Villasana Y, Zamudio-Ochoa A, Shingu-Vazquez M, Torres-Larios A and Perez-Martinez X: The pentatricopeptide repeats present in Pet309 are necessary for translation but not for stability of the mitochondrial COX1 mRNA in yeast. J Biol Chem 283: 1472-1479, 2008.

34. Guo C, Ding J, Yao L, et al: Tumor suppressor gene Runx3 sensitizes gastric cancer cells to chemotherapeutic drugs by downregulating Bcl-2, MDR-1 and MRP-1. Int J Cancer 116: 155-160, 2005. 